\title{
Three-Dimensional Observation of Dopant Atoms in Quantitative Scanning Transmission Electron Microscopy
}

\author{
Jinwoo Hwang ${ }^{1}$, Jack Y. Zhang ${ }^{1}$, Adrian J. D’ Alfonso ${ }^{2}$, Leslie J. Allen ${ }^{2}$, and Susanne Stemmer ${ }^{1}$ \\ 1. Materials Department, University of California, Santa Barbara, California 93106-5050, USA \\ 2. School of Physics, University of Melbourne, Victoria 3010, Australia
}

One of the ultimate goals in electron microscopy is 3-dimensional (3D) atomic scale information. The main challenge in obtaining 3D information in microscopy originates from the limited depth resolution. The depth resolution of scanning transmission electron microscopy (STEM) remains above $\sim 10 \mathrm{~nm}$, even with state-of the-art STEM with aberration correction [1]. Through-focal series and confocal approaches can improve the resolution to about $5 \mathrm{~nm}$, which is still not sufficient for atomic scale 3D studies. An alternative approach is to extract dopant depth information based on the STEM image intensity. The atomic number difference between the dopant and the host crystal will alter the intensity of the atomic column that contains the dopant. This approach, however, faces a few important challenges. Probe channeling and dynamical scattering along the depth direction can significantly complicate the dopant information [2], and sets a limit on the usable TEM sample thickness [3]. Furthermore, criteria that define the dopant visibility in the image must be established. To tackle these issues, and test the practical feasibility, a fully quantitative experiment in STEM is required.

In this work, we present the 3D imaging of individual dopants based on quantitative STEM [4]. Quantitative STEM uses absolute scattered intensities from the sample that can be directly compared to simulations [5]. We advance this technique to extract the number and depth location of Gd dopants in the $\mathrm{Sr}$ column of $\mathrm{SrTiO}_{3}$. For the non- $C_{\mathrm{s}}$-corrected probe that we used in this study, the probe channeling along the depth direction in $\mathrm{SrTiO}_{3}$ shows the first maximum at a thickness of $4 \mathrm{~nm}$, which therefore becomes the practical limit of usable sample thickness for unambiguous interpretation [Fig. 1(a)]. We use column-averaged intensities to avoid the dependence on difficult-to-determine parameters, such as the effective source size and instability of the instrument [6]. Using the average intensity of Ti-O columns surrounding a Sr column as the local thickness reference, we quantified the experimental error from the undoped $\mathrm{SrTiO}_{3}$, which defines the practical dopant visibility limit in the doped $\mathrm{SrTiO}_{3}$.

The intensities of the Sr columns that contain at least one Gd dopant are directly compared to simulated image intensities. Since the experimental data points are scattered from the simulated (ideal) data points due to the experimental error, the quantified experimental error was taken into account for the calculation of the probability of the dopant depth position. From the probability, we calculated the expectation value with an uncertainty in the depth position of the dopant for each column. As an example, Fig. 1(b-e) shows a Gd dopant cluster that was characterized in $\mathrm{SrTiO}_{3}$, with the most probable dopant depth positions indicated with the red atoms ( $\mathrm{d}$ and e). A very high confidence level of dopant depth is achieved, as the depth uncertainty is smaller than one $\mathrm{SrTiO}_{3}$ unit cell $(0.39 \mathrm{~nm})$ [4]. Our work demonstrates an important practical application of quantitative STEM, and shows great promise for the 3D structural determination of nanomaterials with a wide range of compositions [7].

\section{References}

[1] H. L. Xin and D. A. Muller, Microsc. Microanal. 16, 445 (2010).

[2] P. M. Voyles et al., Microsc. Microanal. 10, 291 (2004). 
[3] A. Mittal and K. A. Mkhoyan, Ultramicroscopy 111, 1101 (2011).

[4] J. Hwang et al., Phys. Rev. Lett. 111, 266101 (2013).

[5] J. M. LeBeau, et al., Phys. Rev. Lett. 100, 206101 (2008).

[6] H. E et al., Ultramicroscopy 133, 109 (2013).

[7] This work is supported by DOE (DEFG02-02ER45994).
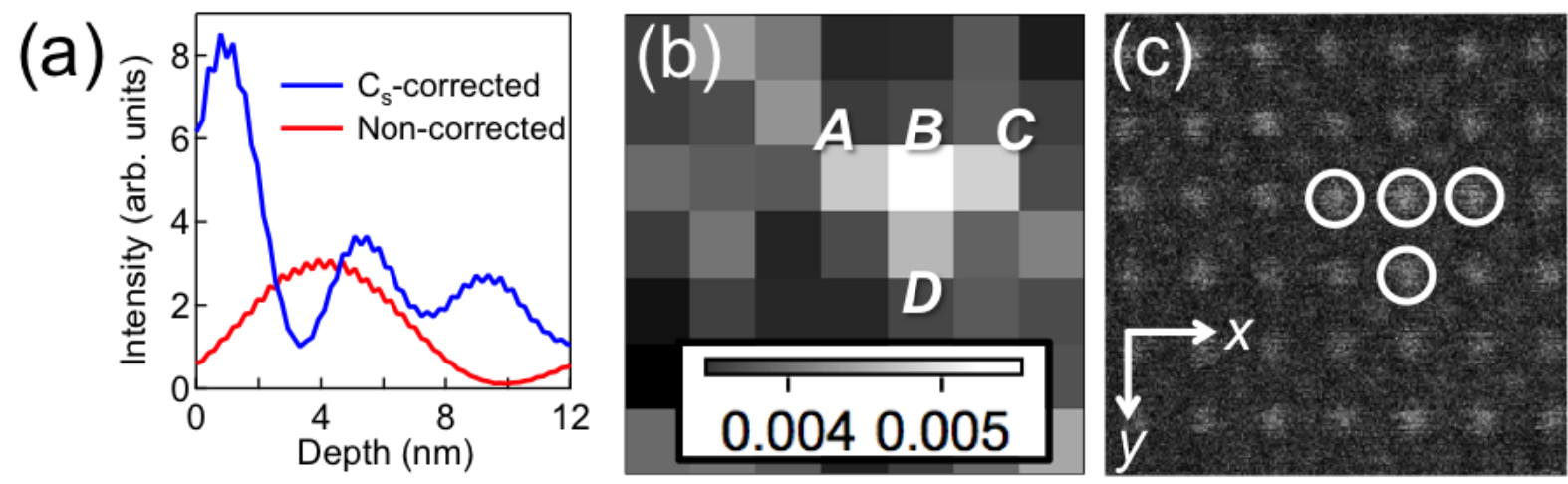

(e)

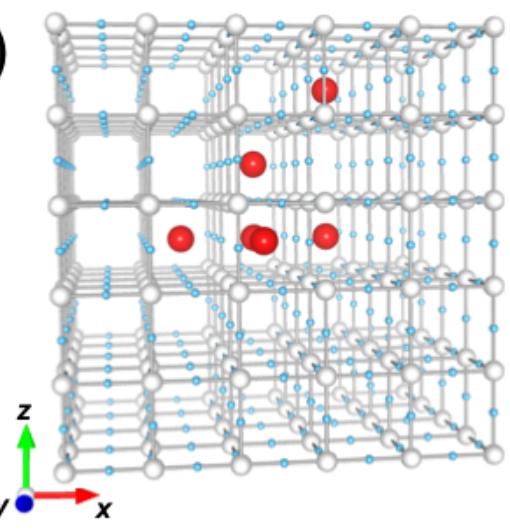

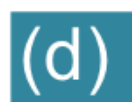

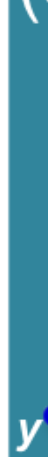

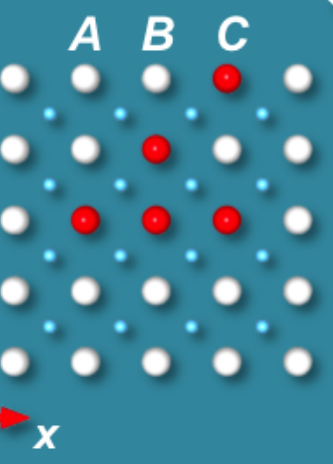

Beam

$100 \%$

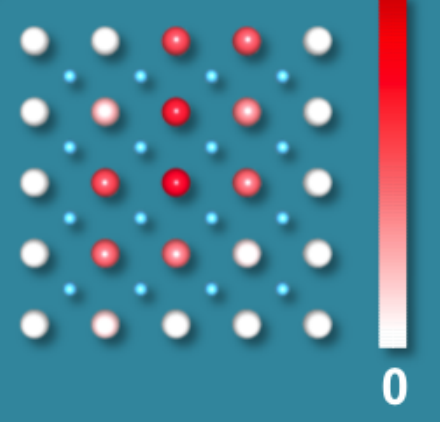

Fig. 1. (a) Probe channeling characteristics for $C_{S}$-corrected and non-corrected probes. The probe is positioned above a $\mathrm{Sr}$ column in $\mathrm{SrTiO}_{3}$, along 100. A non-corrected probe (convergence angle $=9.6$ mrad, $300 \mathrm{kV}$ ) was used in this study. (b-e) 3D imaging of individual Gd dopants in $\mathrm{SrTiO}_{3}$ using quantitative STEM. (b) Column intensity map of Sr columns of the region shown in the quantitative STEM image in (c). The bright columns containing dopants AD form a dopant cluster. (d) Slice containing columns A-C in (b) for (left) the most probable Gd positions and (right) the probability. (e) 3D map of the most probable Gd dopant locations for columns A-D. Sr atoms are not shown and some $\mathrm{Ti}$ and $\mathrm{O}$ atoms were removed for clarity [4]. 